\title{
Efecto de la falta de acuerdos arancelarios en exportaciones de atún Ecuatoriano a la Unión Europea: análisis de tendencia en el período 2010 - 2015
}

\section{Effect of the lack of tariff agreements on exports of Ecuadorian tuna to the European Union: trend analysis in the 2010-2015 period}

Jorge Andrés Izaguirre Olmedo

Instituto Superior Tecnológico Espíritu Santo, Ecuador

María Antonia Benavente González

Instituto Superior Tecnológico Espíritu Santo, Ecuador

Autores para correspondencia: jorgeandres.izaguirre@gmail, mabenavente@ outlook.com Fecha de recepción: 21 de Febrero de 2017 - Fecha de aceptación: 28 de Abril de 2017

\section{Resumen}

Este trabajo de investigación realiza un análisis de tendencia de las exportaciones de atún del Ecuador a la Unión Europea (UE), considerando el período en el que el país no formó parte de los beneficiarios del Sistema Generalizado de Preferencias (SGP) de la UE. El análisis se realiza en cifras de exportación trimestrales proporcionadas por la Aduana del Ecuador. El resultado esperado es un quiebre en tendencia, producto de un encarecimiento del atún ecuatoriano; sin embargo, este resultado no explica en sí mismo toda la problemática del sector.

Palabras Claves: atún; SGP; exportación; tipo de cambio

\begin{abstract}
This investigation work realizes an analysis of trend about the exportations of Ecuadorian tuna to the European Union (EU), taking into consideration the period that Ecuador was not part of the list of beneficiaries of the Generalized System of Preferences of the EU. The analysis is made with quarterly export data that were provided from the Customs of Ecuador. The expected result is a change of trend, as a result of increasing of the price of Ecuadorian tuna; however, this result does not explain all the limitations of the industry.
\end{abstract}

Key words: tuna; GPS; exports; exchange rate 


\section{Introducción}

El Ecuador es un país productor y exportador de bienes primarios, destacándose en industrias petrolera, bananera, atunera, florícola, cacaotera, camaronera, entre otras. Según el Instituto de Promoción de Exportaciones e Inversiones del Ecuador (PROECUADOR), las bondades del clima favorecen la producción de una gran variedad de especies de flora y fauna. (Dirección de Inteligencia Comercial e Inversiones, 2013)

Muchos son los factores que pueden incidir en la expansión o contracción de la demanda de un producto como el atún ecuatoriano. Así mismo, diversos son los factores que inciden sobre desplazamientos de la oferta. Ambos cambios afectan tanto al precio de un producto en el mercado, como a la producción y venta del mismo. Entre los factores referenciados se pueden considerar la aparición de nuevos competidores, condiciones naturales, crisis financieras de países importadores, barreras arancelarias, entre otros (Bueno, 1994).

El atún forma parte de los productos tradicionales de exportación del Ecuador y uno de sus principales destinos es la Unión Europea. Según el Econ. Ángel Cedeño, experto del sector, entrevistado en 2016, existen cuatro factores fundamentales que afectaron al sector atunero desde su crecimiento acelerado a partir del 2008 hasta su caída en los últimos dos años: la caída del precio del petróleo, la crisis financiera, la elevación de costos para obtención del pescado y la eliminación de las preferencias arancelarias SGP a partir del 2014 (Taiano, 2016).

Durante el 2016, Ecuador ha vuelto a formar parte de los países firmantes del acuerdo SGP con la Unión Europea, obteniendo así una serie de beneficios arancelarios, específicos para cada tipo de producto, tanto en exportaciones como en importaciones, que se irán aplicando paulatinamente. No obstante, durante el periodo que Ecuador estuvo excluido del acuerdo, se generó una reducción de ventas hacia el bloque de la UE debido al encarecimiento del producto, por la aplicación de medidas arancelarias. Esta problemática no se verá superada se forma inmediata por la firma del tratado debido a la pérdida de participación en el mercado que sufrieron los exportadores. El objeto del presente estudio es analizar el efecto de la falta de acuerdos arancelarios en exportaciones de atún ecuatoriano a la unión europea, tomando como periodo de estudio del $2010-2015$.

A pesar de que la problemática analizada en el presente estudio ya ha sido superada, la investigación se justifica en la cuantificación del efecto positivo sobre el mercado que se alcanza al negociar los acuerdos con la Unión Europea. Esta cuantificación permitirá a los decisores de políticas económicas determinar el grado de importancia de seguir apoyando los acuerdos bilaterales y las negociaciones de libre comercio.

\section{Metodología}

La investigación tiene un enfoque cuantitativo y utiliza series temporales. Para las estimaciones se utilizaron datos de exportación de atún en dólares a la Unión Europea desde el 2010 hasta finales del 2015, proporcionados por la Aduana del Ecuador; así como otros datos del Banco Central del Ecuador sobre las exportaciones totales de atún, para un análisis previo más general. Debido a la volatilidad que presenta la serie, se trabajó la data en trimestres. 
El análisis se realiza de forma gráfica y analítica. Para la forma gráfica se elaborará un diagrama de dispersión con un punto de quiebre a finales del 2013. Para la parte analítica se estimará una regresión por mínimos cuadrados ordinarios utilizando una variable ficticia al cierre del 2013 y una variable ficticia iterativa para obtener mayor información de la serie.

Como limitación del estudio se debe reconocer que el análisis planteado sólo muestra el comportamiento de la variable exportaciones de atún en tendencia, y en ningún momento tiene como objetivo explicarla, puesto que al hacerlo se requeriría considerar en el estudio otras variables independientes, tales como: tipo de cambio, oferta de nuevos mercados, precio internacional, precio del barril de crudo, costos de producción, entre otros; y estaría fuera del objetivo del presente estudio.

\section{Revisión de la Literatura}

Considerando que el atún es uno de los principales productos de exportación del Ecuador, existen diversos estudios realizados sobre la evolución de las exportaciones del mismo, problemas que afectan a la industria a nivel local e internacional y efectos de su desarrollo sobre la economía del país (Proecuador, 2016).

En el estudio realizado por Prieto (2009) se aborda el desarrollo de la industria pesquera del atún en el Ecuador a través del impacto de los subsidios en su sustentabilidad y en el comercio. Dicho estudio concluye que se requiere de subsidios por parte del gobierno para un crecimiento más acelerado del sector. En el caso de Ecuador, los subsidios, proporcionalmente hablando, son menores que en otros países exportadores de atún, citando a modo de ejemplo el caso de Tailandia que recibió importantes subsidios pesqueros y pudo ingresar a la Unión Europea con precios muy competitivos. Así mismo indica que "la ayuda internacional es fundamental para que los países con menores recursos fiscales puedan cumplir los criterios mínimos de sustentabilidad propuestos para una administración pesquera efectiva." (Prieto, 2009, pág. 107). Esta ayuda incluye beneficios arancelarios como los que se consiguieron para Ecuador con el SGP, tema de estudio en la presente investigación.

Calderón (2014) estudió la sustentabilidad de la industria atunera ecuatoriana en el período 2014 - 2020, para lo cual analizó la serie de exportaciones del 2000 al 2013 y revisó las variables que incidían sobre su evolución. En su investigación concluye que el auge de los commodities y productos primarios ha terminado y que en el 2020, las exportaciones del atún del Ecuador deberían alcanzar un aproximado de 2500 millones de dólares. Concluye también que el nivel de capturas se ha incrementado, al igual que la capacidad de almacenamiento. Sin embargo el análisis técnico abarca del año 2000-2013, y por tanto no considera el hecho de que Ecuador haya dejado de beneficiarse del SGP en el año 2014.

En la tesis de grado elaborada por J. Barre (2015) se analiza la incidencia de la exportación del atún ecuatoriano a los principales mercados internacionales, identificando, a partir de un análisis FODA de la industria atunera del país, cuáles son los principales inconvenientes para el desarrollo del sector: insuficiente nivel de capturas, embarcaciones con poca capacidad de almacenamiento, falta de inversión y competencia agresiva en precios por 
parte de otros países, en especial Tailandia. En su investigación se realiza una revisión de la evolución de las exportaciones de atún a la Unión Europea del 2010 al 2014 y se enfoca, principalmente, en la descripción anual de los datos y la investigación cualitativa sobre las causas que pueden determinar la desaceleración observada (Barre, 2015). No profundizó en análisis técnico al respecto, dejando un buen punto de partida para la investigación desarrollada en este trabajo.

Rugel (2015) estudia el impacto de la aplicación del SGP sobre el camarón ecuatoriano y su ingreso a la UE. Al momento del estudio, el camarón pagaba $18 \%$ de arancel y el atún $24 \%$. En su investigación indica que el $12 \%$ de las exportaciones ecuatorianas son a la UE y que el SGP incrementaría la competitividad del país en los mercados, lo que permitiría mejorar niveles de rentabilidad, producción y empleo en el sector (Rugel, 2015). El estudio sirve a esta investigación pues demuestra la importancia que tiene para Ecuador la pertenencia al SGP.

\section{Resultados}

Para el estudio específico de exportación de atún a la Unión Europea, según se indicó en la metodología, se utilizaron datos de la Aduana del Ecuador. Sin embargo, el Banco Central del Ecuador ofrece también cifras de exportaciones de atún, aunque sin reflejar el país de destino. La figura 1 muestra la evolución de las exportaciones totales del atún ecuatoriano desde enero del 2010 hasta diciembre del 2015 en miles de dólares FOB.

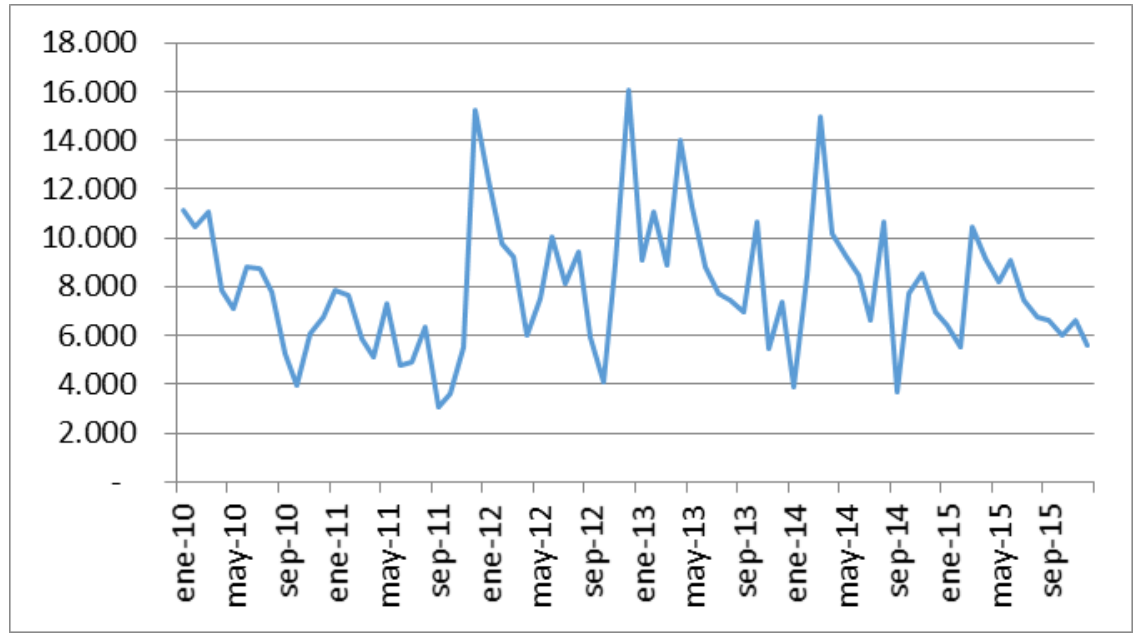

Figura 1

Exportaciones de Atún del Ecuador

Fuente: Banco Central del Ecuador

Como puede observarse en la gráfica, existe una alta volatilidad en las cifras con períodos claros de estacionalidad alta y baja; por ello, con una simple observación sería difícil determinar una tendencia creciente o decreciente en la serie. Las cifras de exportaciones por años se muestran en la tabla 1 .

Tabla 1 Exportaciones Anuales de Atún en el Ecuador

\begin{tabular}{ll}
\hline Años & Exportaciones \\
\hline $\mathbf{2 0 1 0}$ & 94.932 \\
\hline
\end{tabular}




\begin{tabular}{ll}
\hline $\mathbf{2 0 1 1}$ & 77.286 \\
$\mathbf{2 0 1 2}$ & 107.476 \\
$\mathbf{2 0 1 3}$ & 108.611 \\
$\mathbf{2 0 1 4}$ & 99.399 \\
$\mathbf{2 0 1 5}$ & 87.843
\end{tabular}

Fuente: Banco Central del Ecuador

Según se observa en las cifras anuales, las exportaciones empiezan a descender a partir del 2014, coincidiendo con la no vigencia del SGP para Ecuador. En este sentido, acorde a las distintas teorías recogidas en la obra de Bueno (1994), los exportadores ecuatorianos deberían analizar y tratar de incursionar en nuevos mercados que permitan compensar la pérdida de cuota que se generaría en la UE. Sin embargo, este proceso es lento, lo cual se evidencia en las cifras presentadas. Al analizar solamente las exportaciones dirigidas a la Unión Europea, esta diferencia debería verse más marcada.

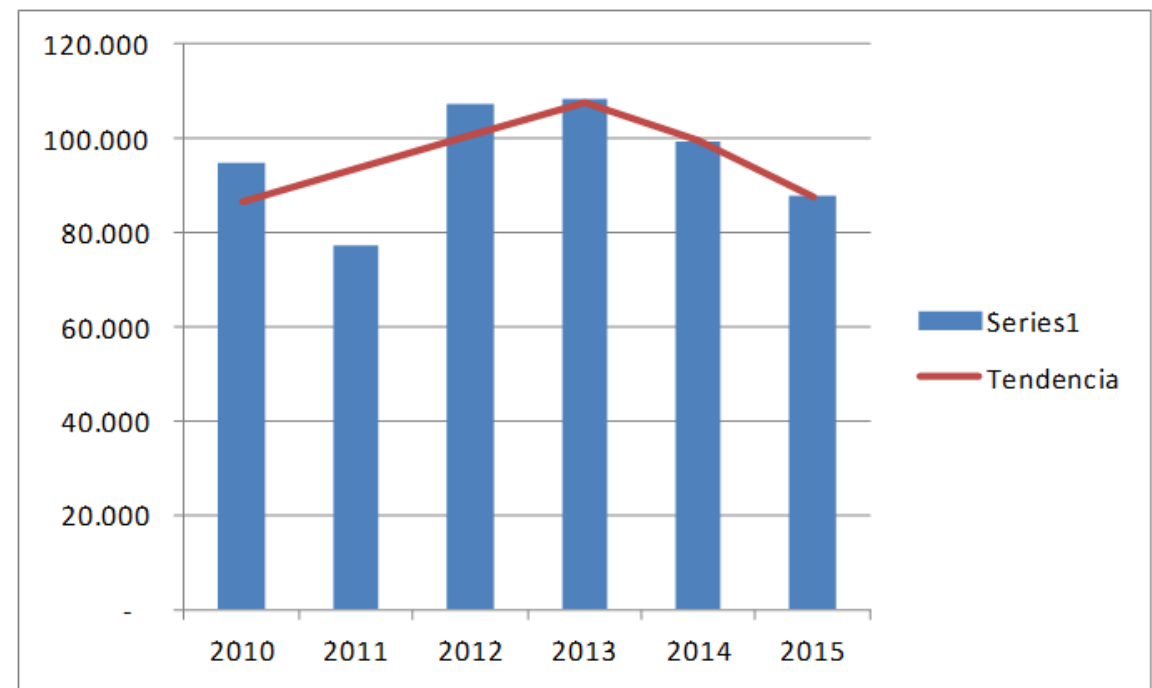

Figura 2: Tendencia de Exportaciones de Atún en el Ecuador Fuente: Banco Central del Ecuador

Como se observa en la figura 2, existe un cambio de tendencia en las exportaciones a partir del año 2014, pasando de pendiente positiva a negativa. En este punto, resultaría de interés analizar los escenarios hipotéticos de predicciones de las exportaciones bajo diferentes supuestos y métodos de predicción.

\section{Análisis Predictivo de la Exportación de Atún del Ecuador}

Existen distintos métodos de predicción que involucran distintos supuestos. Cada modelo se ajusta a la realidad dependiendo de las circunstancias. Para este caso se utilizarán los siguientes métodos: Promedio, Media Móvil de dos períodos, Suavización Exponencial y Tendencia.

Tabla 2: Pronóstico de Exportaciones

\begin{tabular}{llllll}
\hline Años & Exportaciones & Promedio & Media Móvil & Suav Exp & Tendencia \\
\hline
\end{tabular}




\begin{tabular}{llllll}
\hline $\mathbf{2 0 1 0}$ & 94.932 & 95.925 & & & 93.637 \\
$\mathbf{2 0 1 1}$ & 77.286 & 95.925 & & 94.932 & 94.552 \\
$\mathbf{2 0 1 2}$ & 107.476 & 95.925 & 86.109 & 80.610 & 95.467 \\
$\mathbf{2 0 1 3}$ & 108.611 & 95.925 & 92.381 & 102.109 & 96.382 \\
$\mathbf{2 0 1 4}$ & 99.399 & 95.925 & 108.044 & 104.265 & 97.297 \\
$\mathbf{2 0 1 5}$ & 87.843 & 95.925 & 104.005 & 98.699 & 98.212 \\
$\mathbf{2 0 1 6}$ & & 95.925 & 93.621 & 90.888 & 99.127 \\
\hline
\end{tabular}

Fuente: Banco Central del Ecuador

La tabla 2 muestra los resultados de los pronósticos a través de los métodos indicados. Según método del promedio, en 2016 las exportaciones debieron ser de 95,925 miles de dólares americanos (USD). El promedio considera la misma probabilidad de ocurrencia o peso a todos los datos, por lo que el resultado no recogerá las alteraciones o shocks que pueda sufrir el mercado.

La media móvil utilizada, calculada con dos períodos, se muestra un poco inferior al promedio. Al compararse con éste, la media móvil se vuelve un poco más confiable, puesto que recoge la información más reciente proporcionada por los datos. Caso similar sucede con los otros dos métodos de predicción. En este sentido, la suavización exponencial corrige la predicción en base al error registrado en los períodos anteriores; y la tendencia calcula un promedio condicionado al comportamiento de la serie o su evolución (Hoel \& Jessen, 1983).

Para la elección de un método de predicción, de entre los indicados, se debe recurrir a algún mecanismo de verificación de ajuste. Para esto se procede a utilizar el método de la suma de errores absolutos, en el cual en primer lugar se obtienen los errores de cada estimación, después se aplica el valor absoluto para eliminar diferencias de signos, y por último se selecciona aquel valor con menor suma de error absoluto. Los resultados se reflejan en la tabla 3.

Tabla 3: Comparación de Errores en Valores Absolutos

\begin{tabular}{llllll}
\hline Años & Exportaciones & Promedio & $\begin{array}{c}\text { Media } \\
\text { Móvil }\end{array}$ & $\begin{array}{l}\text { Suav } \\
\text { Exp }\end{array}$ & Tendencia \\
\hline 2012 & 107.476 & 11.552 & 21.367 & 26.866 & 12.009 \\
2013 & 108.611 & 12.687 & 16.230 & 6.502 & 12.229 \\
2014 & 99.399 & 3.475 & 8.645 & 4.866 & 2.102 \\
2015 & 87.843 & 8.082 & 16.162 & 10.856 & 10.369 \\
Total & & 35.794 & 62.404 & 49.090 & 36.709 \\
\hline
\end{tabular}

Fuente: Banco Central del Ecuador

Como puede observarse en la tabla 3, el mecanismo de predicción de mejor ajuste utilizando el método de la suma de errores absolutos es el promedio, considerando el periodo 2012 a $2015^{1}$; seguido por la tendencia. No obstante, es necesario considerar que ninguno de los

\footnotetext{
${ }^{1}$ Se excluyen los primeros dos períodos por no existir información para todos los predictores por igual.
} 
predictores fue condicionado a la inexistencia del acuerdo con la Unión Europea, lo que afectaría a la serie desde finales del 2013 hasta el final del período de análisis. Sin embargo esto se considera en el análisis específico de las exportaciones de atún a la Unión Europea.

\section{Las Exportaciones de Atún a la Unión Europea}

Una vez analizadas las cifras de exportaciones totales del producto, la investigación se enfoca en la evolución de las exportaciones a la Unión Europea. De acuerdo a lo indicado en la metodología, las cifras de exportaciones totales fueron obtenidas de la página del BCE, más dicha fuente no permite sectorizar por destino dichas exportaciones. En esta sección se trabaja con datos de la Autoridad Aduanera del Ecuador, los cuales se calcularon de manera trimestral para suavizar la serie.

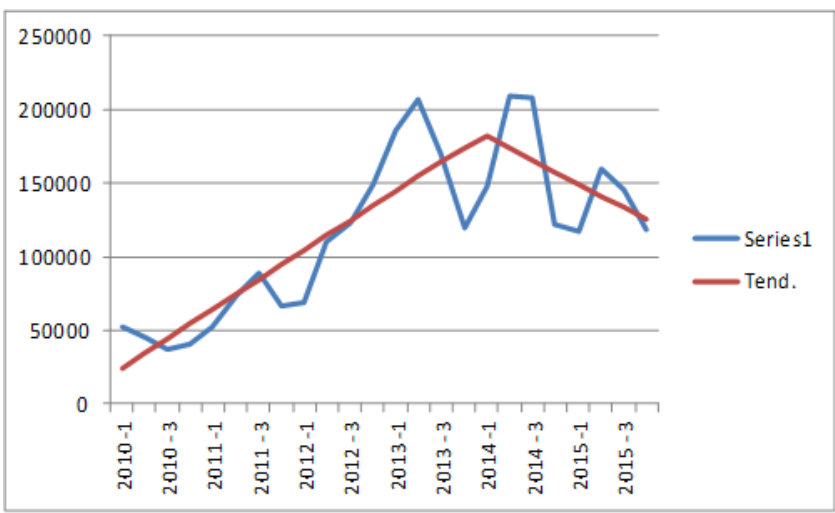

Figura 3: Exportaciones de Atún a la Unión Europea y tendencia Fuente: Aduana del Ecuador

Como puede observarse en la figura 3, hasta mediados del 2013 se observa un crecimiento de la serie. A partir de ese punto, la tendencia se revierte. No obstante, aunque el objetivo de este trabajo es verificar si existe un cambio de tendencia y la magnitud del mismo, éste no es evidencia suficiente para concluir que la no existencia del acuerdo para el Ecuador haya sido la razón de dicho cambio en la serie. Existen otros factores que afectan a las exportaciones y que no son parte de este estudio. En este sentido, aunque el análisis técnico concluya un cambio en tendencia, no se podría asegurar que éste es debido a la no pertenencia del Ecuador al SGP; sin embargo se podría indicar que desde el 2014, en que Ecuador dejó de verse beneficiado del acuerdo, las exportaciones de atún a la UE cambiaron de tendencia.

\section{Análisis Técnico}

Para el análisis técnico se ha realizado una regresión lineal tomando como variable dependiente las exportaciones de atún ecuatoriano realizadas a la unión europea con frecuencia trimestral, desde el primer trimestre del 2010 hasta el cuarto trimestre del 2015. Con el objetivo de testear el cambio en tendencia, como variables independientes se han utilizado dos variables ficticias:

- Dummy de nivel: Mide la diferencia entre el promedio de ambos períodos. Toma el valor de 1 para el período de 2010 hasta finales de 2013 y el valor de 0 para los otros 
casos. En este sentido, el coeficiente indicará cuánto mayor o menor es el promedio de las exportaciones durante el período en el que el Ecuador se estuvo beneficiando del SGP frente al período en el que no fue así.

- Dummy de tendencia: Resulta de multiplicar la tendencia por la dummy de nivel. Así puede determinarse si existe diferencia en el ritmo de crecimiento de la variable durante los períodos indicados.

La siguiente tabla muestra el coeficiente de ajuste del modelo y el análisis de varianza del mismo:

Tabla 4: Estadísticas de la Regresión

\begin{tabular}{ll}
\hline Regresión & \\
Coeficiente de correlación múltiple & 0,83769251 \\
Coeficiente de determinación $\mathbf{R}^{\wedge} \mathbf{2}$ & 0,70172874 \\
$\mathbf{R}^{\wedge} \mathbf{2}$ ajustado & 0,67332196 \\
Error típico & 31594,8975 \\
Observaciones & 24 \\
\hline
\end{tabular}

Tabla 5: Análisis de Varianza

\begin{tabular}{llll}
\hline & Grados de libertad & F & Valor crítico de F \\
\hline Regresión & 2 & 24,7028556 & $3,04383 \mathrm{E}-06$ \\
Residuos & 21 & & \\
Total & 23 & & \\
\hline
\end{tabular}

La tabla 4 muestra un coeficiente $\mathrm{R}$ cuadrado ajustado de 0,67 , lo cual implica que las variables ficticias explican casi el 70\% de las variaciones en la serie. Así mismo el análisis de varianza reflejado en la tabla 5 , arroja un valor inferior al 0.05 , lo que concluye que el modelo si explica a la variable dependiente. La tabla 6 muestra el resultado de los coeficientes de las variables para el análisis de regresión.

Tabla 6: Resultados de la Regresión

\begin{tabular}{ccc}
\hline & Coeficientes & Probabilidad \\
\hline Intercepción & 153300,3901 & $5,9005 \mathrm{E}-12$ \\
Dummy de nivel & $-138663,2498$ & $7,4404 \mathrm{E}-07$ \\
Dummy de tendencia & 9979,159938 & $8,8214 \mathrm{E}-06$ \\
\hline
\end{tabular}

Según se observa en la tabla 6, ambas variables resultan significativas al 1\%. En la dummy de nivel se observa que el coeficiente tiene un valor negativo, lo cual implica que el promedio registrado en el período 2010 - 2013 es menor que el promedio registrado en el período 2014 - 2015. En primera instancia este resultado iría en contra de lo planteado para este estudio; sin embargo, dicho resultado se debe a que durante los primeros trimestres de la serie se observa una tendencia creciente que arranca debido a la oportunidad que los exportadores encuentran en los beneficios arancelarios con la UE. En contraste, a partir del 2014 inicia una reducción paulatina de la serie, que en ese punto suponen el triple de las exportaciones registradas al inicio del 2010. El análisis debe completarse con la revisión del coeficiente de la otra variable. 
En el caso de la dummy de tendencia, el coeficiente es positivo y significativo, lo cual indica que en promedio, durante el período que Ecuador formaba parte del SGP, cada trimestre las exportaciones crecían en 9,979 miles USD más de lo que crecen período a período después del 2014. No obstante, se recomienda analizar únicamente el signo del coeficiente y su significancia debido a que a partir del 2014 la tendencia es negativa, por lo que el coeficiente no debe tener interpretación directa.

\section{Conclusiones}

Según se pudo observar en la sección de resultados, la no pertenencia de Ecuador al Sistema Generalizado de Preferencias tuvo una consecuencia significativa en el nivel de exportaciones de atún hacia el bloque de la Unión Europea. Este nivel de exportaciones detuvo su ritmo de crecimiento, el cual se volvió negativo.

Los factores que inciden sobre el nivel de exportaciones de atún son diversos, y según se mostró en la revisión de la literatura, éstos son internos o externos. Por esta razón la permanencia de Ecuador en el acuerdo con la UE reviste particular importancia. Las ventajas aduaneras y comerciales de las cuales se ve beneficiado el país logran compensar en parte los problemas evidenciados en la revisión de los trabajos previos.

Tomando en consideración lo anterior, se recomienda para una próxima investigación encontrar aquellas variables determinantes del nivel de exportaciones del producto ecuatoriano. Según investigaciones cualitativas, los factores podrían ser el tipo de cambio, precio del barril de petróleo, costo de captura y producción, costos indirectos, entre otros; los cuales deberían intentar testearse como determinantes de la variable dependiente.

\section{Bibliografía}

Banco Central del Ecuador. (s.f.). Recuperado el Julio de 2016, de http://contenido.bce.fin.ec/documentos/PublicacionesNotas/Catalogo/IEMensual/metodologia /nm-bpe.pdf

Banco Central del Ecuador. (2016). Estadísticas Macroeconómicas Presentación Coyuntural.

Barre, J. (2015). La exportación del atún ecuatoriano a los principales mercados internacionales y su incidencia en las principales variables macroeconómicas del Ecuador en el período 2010 2014. Universidad de Guayaquil.

Bueno, E. (1994). Fundamentos de Economía y Organización Industrial. Mc Graw Hill.

Calderón, P. (2014). La sustentabilidad de las Exportaciones de Atún Ecuatoriano: 2014 - 2020. Universidad de Guayaquil.

Comercio, D. E. (20 de Junio de 2014). La captura del atún fortalece al sector pesquero ecuatoriano. Obtenido de http://www.elcomercio.com/actualidad/captura-atun-pesca-ecuador-cifras.html

Dirección de Inteligencia Comercial e Inversiones. (2013). Análisis del Sector Pesca. Quito: Proecuador. 
Franklin, E., \& Gómez, G. (2002). Organización y Métodos: Un Enfoque Competitivo. Mc Graw Hill.

Hoel, P., \& Jessen, R. (1983). Estadística Básica para Negocios y Economía. CECSA. Juncker, J.-C. (s.f.). La web Europa. Obtenido de http://europa.eu/about-eu/basicinformation/about/index_es.htm

Larrucea, J. A. (2002). La Unión Europea, la Comunidad Europea y el derecho comunitario. Sevilla: Universidad de Sevilla.

Mariscal, C. (2001). Formulación y Evaluación de Proyectos. ESPOL.

Ministerio de Comercio Exterior. (2016). Comercio Exterior. Recuperado el Julio de 2016, de www.comercioexterior.gob.ec/sistema-general-de-preferencias

Montero, J. (2015). Análisis de las Exportaciones del Sector Atunero frente a la Eliminación de la Ley de Preferencias Arancelarias Andinas y Erradicación de las Drogas ATPDEA 2009 - 2014. Universidad Agraria del Ecuador.

Prieto, I. (2009). Impacto de los Subsideios Pesqueros en la Sustentabilidad y el Comercio del Atún en el Ecuador. Guayaquil.

PROECUADOR. (2016). Proecuador. Recuperado el Junio de 2016, de http://www.proecuador.gob.ec/

Rafful, R. A. (1996). La Unión Europea: una experiencia de integración regional. Madrid: Plaza y Valdés.

Ross, S., Westerfield, R., \& Bradford, J. (2010). Fundamentos de Finanzas Corporativas. Mc Graw Hill.

Roy, J. \&. (2001a). Las relaciones exteriores de la Unión Europea. Madrid: Plaza y Valdes.

Rugel, S. (2015). Aplicación del Sistema Generalizado de Preferencias Plus para el Ingreso del Camarón Ecuatoriano al Mercado de la Unión Europea. Machala: Universidad Técnica de Machala.

Secretaría Nacional de Planificación y Desarrollo. (2013). Plan Nacional de Desarrollo / Plan Nacional del Buen Vivir 2013 - 2017.

Soler, D. (2008). Diccionario de Logística. Editorial Marge Books.

Taiano, D. (2016). Análisis de las Exportaciones del Sector Atunero del Ecuador hacia la Unión Europea en el período 2010 - 2015. Universidad Católica Santiago de Guayaquil.

Unión Europea. (s.f.). La Unión Europea. Recuperado el Julio de 2016, de http://europa.eu/abouteu/basic-information/about/index-es.htm

Vicioso, T. B. (2016). PMAR Ámbito Lingüístico y Social II. Editex. 